\title{
Indicadores del decaimiento en bosques de Nothofagus pumilio en el norte de la Patagonia, Argentina
}

\section{Indicators of forest decline for Nothofagus pumilio in northern Patagonia, Argentina}

\author{
Milagros Rodríguez-Catón ${ }^{{ }^{*}}$ y Ricardo Villalbal
}

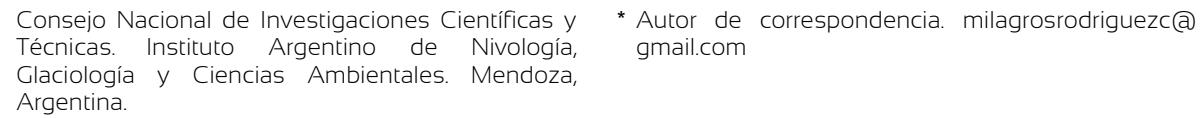

\section{RESUMEN}

El decaimiento forestal está frecuentemente asociado a la mortalidad parcial o total de las copas en un alto porcentaje de individuos de un rodal. Si bien el decaimiento ha sido documentado a escala global, las relaciones entre las condiciones externas de los árboles y su crecimiento radial raramente han sido exploradas. El presente trabajo relaciona la intensidad del decaimiento con el crecimiento radial en 294 árboles de Nothofagus pumilio en el norte de la Patagonia. Los indicadores externos del decaimiento analizados fueron la mortalidad de la copa, la sanidad de la corteza, la incidencia de insectos barrenadores y pájaros carpinteros, así como la presencia de plantas hemiparásitas, hongos y líquenes. Los resultados indican que elevados porcentajes de mortalidad de copa están significativamente relacionados con la disminución del crecimiento radial promedio, siendo esta relación más notoria cuando se usa como medida del crecimiento radial el incremento en área basal en lugar del ancho de los anillos. El deterioro de la corteza y la abundancia de cavidades producto de las actividades de insectos barrenadores y de pájaros carpinteros también estuvieron significativa e inversamente relacionados con el crecimiento. Por el contrario, el crecimiento y la presencia de hemiparásitas, hongos o líquenes no mostraron relaciones significativas. A partir de estos resultados, se propone utilizar en la estimación de la sanidad forestal los indicadores externos (1) mortalidad de copa, (2) deterioro de la corteza y (3) cavidades de insectos y/o pájaros carpinteros, como una medida integral del decaimiento forestal de Nothofagus pumilio en la Patagonia.

PALABRAS ClAVE: anillos de crecimiento, insectos barrenadores, mortalidad de la copa, pájaros carpinteros, sanidad forestal.

\section{ABSTRACT}

Forest decline is associated with partial or total crown mortality in a large percentage of trees at the stand level. While forest decline has been reported on a global context, the relationships between the external conditions of trees and their radial growth have rarely been reported. This study relates the intensity of decline with radial growth in 294 Nothofagus pumilio trees in northern Patagonia. The selected external indicators of decline were crown mortality, bark health, the incidence of boring insects and woodpeckers, as well as the presence of hemiparasite plants, fungi and lichens. High percentages of crown mortality are significantly related to decreasing radial growth of remaining trees. This relationship is more reliable when basal area increments rather than ring widths are used as estimates of radial growth. Bark health and abundance of cavities, resulting from the activities of boring insects and woodpeckers, were also significantly inversely related to growth. In contrast, no statistically significant relationships were found between growth and the presence of hemiparasites, fungi or lichens. Based on these results, we recommend the use of the following external indicators (1) crown mortality, (2) bark conditions and (3) cavities from boring insects and/or woodpeckers, to comprehensively characterize the Nothofagus pumilio forest decline in Patagonia.

KEYWORDS: growth rings, wood-boring insects, crown mortality, woodpeckers, forest health. 


\section{INTRODUCCIÓN}

El decaimiento forestal se asocia con la muerte progresiva de la copa de los árboles que en muchos casos conduce a la mortalidad masiva del bosque (Mueller-Dombois, 1992; Montoya Oliver, 1995; Calí, 1996). Este proceso de deterioro sanitario de los bosques ha sido documentado desde principios del siglo $\mathrm{XX}$, observándose un aumento en la cantidad de estudios durante las últimas décadas (McDowell, Ryan, Zeppel y Tissue, 2013; Williams et al., 2013; Wong y Daniels, 2017). El deterioro de los bosques es evidente cuando la cantidad de individuos muertos o con elevados porcentajes de mortalidad de copa (mayores a 30\%) es comparativamente superior a lo observado en bosques sanos de la misma especie (Kolb \& Mccormick, 1993; Allen et al., 2010). Si bien el decaimiento ha sido ampliamente documentado tanto a escala de rodal (Kolb y Mccormick, 1993; Bowers y Turner, 2005) como regional (Hogg, Brandt y Michaelian, 2008; Vila-Cabrera, Martínez-Vilalta, Galiano y Retana, 2013; Worrall et al., 2013), las evidencias provenientes de distintos bosques y regiones en el mundo indican que se trata de un proceso global con una importante componente climática como agente causal (Ciesla y Donaubauer, 1994; Allen et al., 2010; Steinkamp y Hickler, 2015).

A pesar de haber sido estudiados durante varias décadas, los procesos de senescencia, decaimiento y muerte de los árboles no son del todo comprendidos y los estudios a nivel individuo son fundamentales para su comprensión (Franklin, Shugart y Harmon, 1987; Rice, Matzner, Byer y Brown, 2004; Ireland, Moore, Fulé, Zegler y Keane, 2014). En las últimas décadas se ha observado que la condición externa va acompañada de tasas de crecimiento radial bajas antes de la muerte de los árboles o en ejemplares aún vivos que presentan mortalidad parcial de copa (Amoroso y Larson, 2010; Attis Beltrán, Martínez Pastur, Ivancich, Lencinas y Chauchard, 2013; Suarez, Ghermandi y Kitzberger, 2004). Asimismo, las bajas tasas de crecimiento pueden estar relacionadas a factores climáticos que disparan tendencias negativas en el crecimiento de árboles o rodales que, por su edad, historia o características de sitio, se encuentran predispuestos a ser afectados (Anderegg, Kane y Anderegg, 2013; Cailleret, Nourtier, Amm, Durand-Gillmann y Davi, 2013; Rodríguez-Catón, Villalba, Morales y Srur, 2016).
Este crecimiento radial reducido disminuye el transporte de sustancias por el leño y crea un desbalance fisiológico que impide movilizar el agua requerida por la copa provocando la muerte parcial de las ramas principales (Manion, 2003). A su vez, estos procesos incrementan la susceptibilidad al ataque de plagas, enfermedades $\mathrm{u}$ otros factores que contribuyen o inducen la muerte de los árboles, tal como lo indica el modelo en espiral de eventos en cascada propuesto por Manion (1981, 2003). Si bien el deterioro sanitario de las copas de los árboles ha sido asociado con ritmos lentos de crecimiento, son pocos los estudios que relacionan en forma comprensiva la condición externa de los árboles con los patrones de crecimiento radial (Dobbertin, 2005; Ohno et al., 2008). Por un lado, es necesario conocer estas relaciones para comprender mejor los procesos de decaimiento y mortalidad, pero, a su vez, resulta útil y práctico poder establecer cuáles son los indicadores externos más confiables para estimar la intensidad del decaimiento de un rodal.

En la Patagonia, los individuos con mortalidad parcial de copas son comunes en rodales del género Nothofagus ubicados próximos a la estepa patagónica o en sitios ventosos con suelos poco desarrollados donde la disponibilidad hídrica es reducida (Veblen, Donoso, Kitzberger y Rebertus, 1996). En el norte de la Patagonia, la reducción del crecimiento y la mortalidad masiva de individuos de Austrocedrus chilensis (D. Don) Endl. y Nothofagus dombeyi (Mirb.) Oerst. han sido relacionados con la ocurrencia de sequías extremas (Amoroso y Larson, 2010; Mundo et al., 2010; Villalba y Veblen, 1998; Suarez et al., 2004). Sin embargo, el fenómeno de decaimiento en Nothofagus pumilio (Poepp. et Endl.) Krasser (Nothofagaceae), la especie arbórea más ampliamente distribuida en la Patagonia, ha recibido escasa atención. Estudios recientes indican que el inicio del decaimiento observado en extensas áreas de la distribución de $N$. pumilio está asociado a eventos de pronunciadas sequías (Rodríguez-Catón et al., 2016). En los mismos sitos, Rodríguez-Catón, Villalba, Srur, y Luckman (2015) observaron que árboles con grados avanzados de mortalidad de copa y follaje reducido coexisten con individuos sanos en un mismo rodal (Fig. 1). 


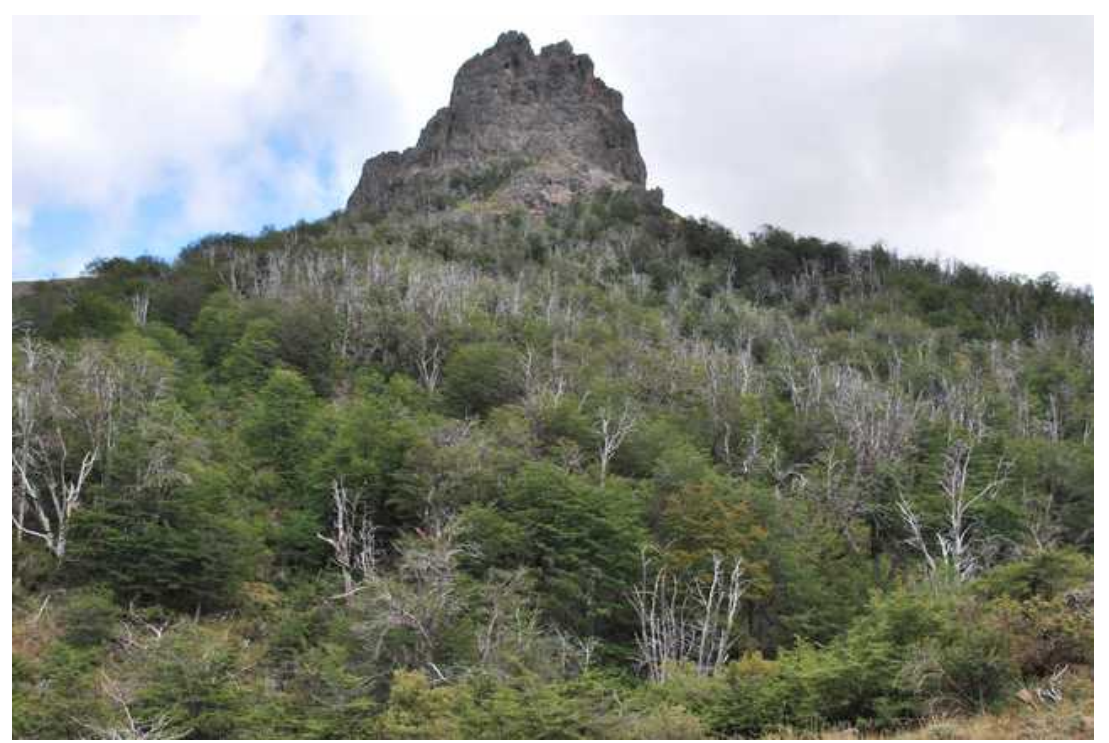

Figura 1. Bosque de Nothofagus pumilio en decaimiento. Paso Córdova (sitios de muestreo C1 y C2, ver Tabla 1 y Fig. 2), Neuquén, Argentina.

TABLA 1. Sitios de muestreo.

\begin{tabular}{|c|c|c|c|c|c|c|c|}
\hline Sitio & Código & Latitud S & Longitud O & $\begin{array}{l}\text { Elevación } \\
\text { (m) }\end{array}$ & Área (ha) & $\begin{array}{l}N \quad \text { vivos } \\
\text { (árboles ha-l) }\end{array}$ & $\begin{array}{l}N \quad \text { total } \\
\text { (árboles ha-l) }\end{array}$ \\
\hline Hualcupen & $\mathrm{HU}$ & $38^{\circ} \mathrm{Ol}^{\prime} \mathrm{OO}$ & $70^{\circ} 56^{\prime} 00^{\prime \prime}$ & 4.8. & 0,2 & 180 & 190 \\
\hline PinoHachado & $\mathrm{PH}$ & 38॰ 39' 30" & $70^{\circ} 51^{\prime} 30 "$ & 1595 & 0,18 & 144 & 177 \\
\hline \multirow[t]{2}{*}{ Cerro Taraya } & $\mathrm{T} 1$ & $39^{\circ} 06^{\prime} 34^{\prime \prime}$ & $71^{\circ} 09^{\prime} 15^{\prime \prime}$ & 1470 & 0,225 & 182 & 204 \\
\hline & $\mathrm{T} 2$ & $39^{\circ} 06^{\prime} 34^{\prime \prime}$ & $71^{\circ} 08^{\prime} 32^{\prime \prime}$ & 1270 & 0,14 & 243 & 329 \\
\hline \multirow[t]{2}{*}{ Paso Córdoba } & $\mathrm{Cl}$ & $40^{\circ} 35^{\prime} 40^{\prime \prime}$ & $71^{\circ} 08^{\prime} 40^{\prime \prime}$ & 1365 & 0,2 & 260 & 330 \\
\hline & $\mathrm{C} 2$ & $40^{\circ} 35^{\prime} 33^{\prime \prime}$ & $71^{\circ} 08^{\prime} 33^{\prime \prime}$ & 1314 & 0,27 & 181 & 270 \\
\hline Cerro Challhuaco & $\mathrm{CH}$ & $41^{\circ} 15^{\prime} 42^{\prime \prime}$ & $71^{\circ} 16^{\prime} 57^{\prime \prime}$ & 1363 & 0,124 & 427 & 467 \\
\hline Perito Moreno & MO & $41^{\circ} 47^{\prime} 42^{\prime \prime}$ & $71^{\circ} 34^{\prime} 18^{\prime \prime}$ & 1230 & 0,189 & 238 & 264 \\
\hline Cerro Piltriquitrón & $\mathrm{PI}$ & $41^{\circ} 55^{\prime} 32^{\prime \prime}$ & $71^{\circ} 28^{\prime} 16^{\prime \prime}$ & 1350 & 0,156 & 199 & 237 \\
\hline \multirow[t]{2}{*}{ Nahuel Pan } & R2 & $43^{\circ} \mathrm{Ol}^{\prime} 5 \mathrm{O}^{\prime \prime}$ & $71^{\circ} 13^{\prime} 34^{\prime \prime}$ & 1047 & 0,15 & 280 & 340 \\
\hline & R3 & $43^{\circ} \mathrm{Ol} 15^{\prime \prime}$ & $71^{\circ} 13^{\prime} 10^{\prime \prime}$ & 1024 & 0,08 & 475 & 600 \\
\hline
\end{tabular}

Para cada sitio se indica su posición geográfica, elevación, área de la parcela muestreada, así como el número de árboles vivos y el total por hectárea. 


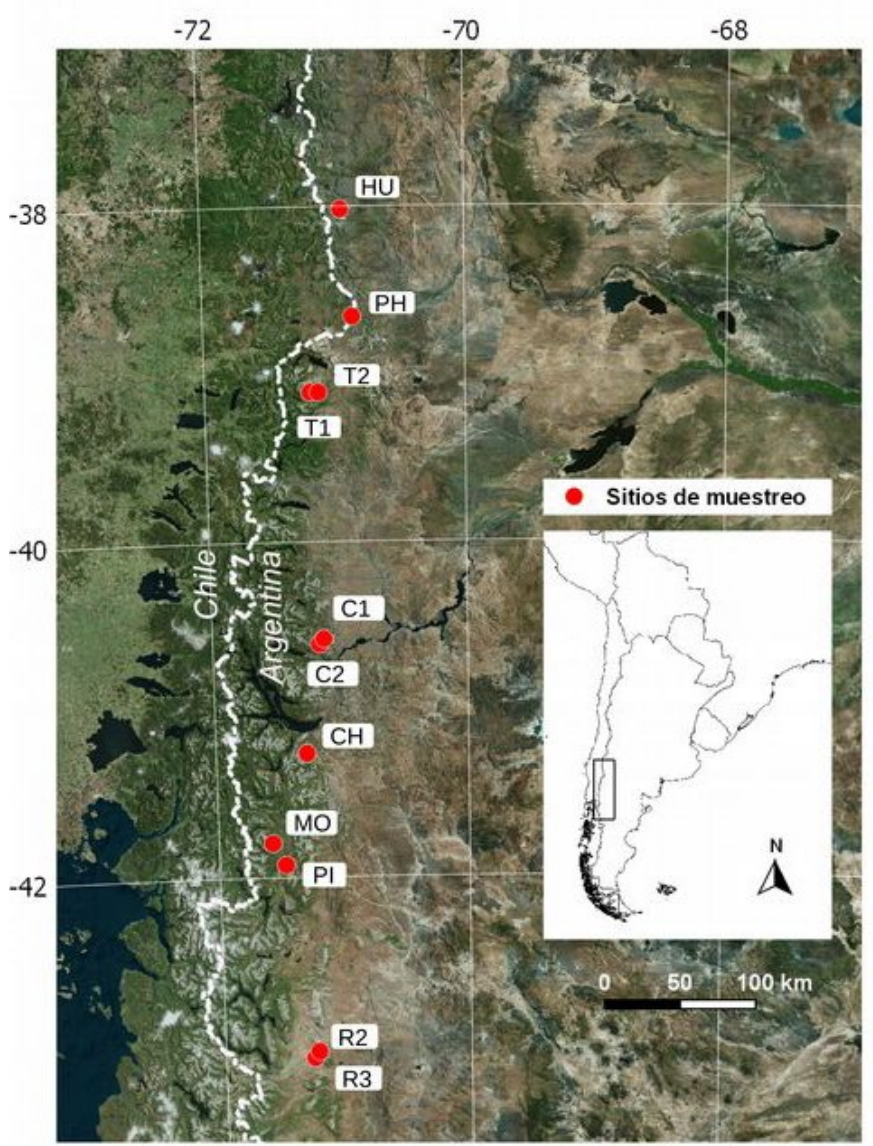

FIGURA 2. Ubicación de los sitios de muestreo a lo largo de un transecto de $500 \mathrm{~km}$ en el norte de la Patagonia argentina. Las parcelas muestreadas están indicadas con círculos rojos e identificadas con los códigos listados en la tabla 1.

Esto demuestra que el decaimiento se manifiesta de forma diferente entre individuos y que existen diferentes intensidades de afectación posibles de ser cuantificadas en un mismo rodal. Estos trabajos muestran que los árboles de mayor tamaño y con mayores porcentajes de mortalidad de copa muestran en su mayoría patrones con tendencias negativas de crecimiento. Sin embargo, hasta el momento no se han comparado de forma detallada el conjunto de posibles indicadores externos de la sanidad de los individuos que representen las tendencias temporales del crecimiento radial.

Estudios previos establecieron relaciones entre el estado sanitario de los bosques de Nothofagus y su crecimiento. Por ejemplo, Henríquez-Velásquez, Henríquez y Aravena (2012) observaron que la presencia de la planta hemiparásita Misodendrum punctulatum (Banks ex DC.) afecta la arquitectura de la copa y produce una reducción en el crecimiento radial de los individuos de N. pumilio. Por su parte, el carpintero negro (Campephilus magellanicus) selecciona árboles de $N$. pumilio con crecimiento reducido y grados avanzados de mortalidad de copa para alimentarse y anidar (Ojeda, 2004; Ojeda, Suarez y Kitzberger, 2007). Entre las diferentes estrategias de alimentación, esta ave realiza excavaciones en los fustes de árboles vivos principalmente, alimentándose de larvas de insectos barrenadores de la madera (Chazarreta, Ojeda y Lammertink, 2012).

A su vez, la incidencia de barrenadores es un predictor importante de la mortalidad en bosques de $N$. dombeyi afectados por eventos extremos de sequías, es decir, rodales con mayor daño por insectos barrenadore,s presentaron un porcentaje más alto de individuos muertos (Suarez et al., 2004). Si bien los hongos parásitos como Cyttaria sp. (llao llao) no afectan significativamente el crecimiento de los árboles afectados, estos parásitos causarían pudriciones posteriormente colonizadas por hongos necrótrofos que aumentan el deterioro de los individuos y su vulnerabilidad a caídas por viento (Frangi et al., 2005). El crecimiento de líquenes foliosos (hongos simbiontes asociados con algas) altera las condiciones de humedad sobre la corteza, facilita la actividad de hongos patógenos y sirve de refugio para diversos insectos que afectan el bosque (Brodo, 1973). También se ha documentado que los tejidos de líquenes fruticosos, como Usnea sp., penetran profundamente en la corteza de los árboles, llegando inclusive hasta el tejido cambial (Brodo, 1973). Si bien no hay evidencias de que la presencia de Protousnea sp. influya sobre el estado sanitario de los árboles en los Andes patagónicos, las partes de un árbol o los árboles con mayor incidencia de luz tendrían un mayor número de talos de Protousnea sp. (Calvelo, Stocker-Wörgöter, Liberatore y Elix., 2005).

En el presente estudio se propone la existencia de relaciones consistentes entre los patrones radiales de crecimiento (indicadores internos), previamente asociados a sequías extremas y la intensidad de los indicadores externos del decaimiento.

\section{OBJETIVOS}

El objetivo de este trabajo fue determinar cuáles de los signos o indicadores externos presentes en individuos de $N$. pumilio son 
más relevantes para cuantificar el decaimiento de un árbol o rodal en forma integrada con los patrones de crecimiento leñoso. Además de la mortalidad de copa, se utilizan otras variables para estimar la condición sanitaria externa de los árboles incluyendo la presencia de plantas hemiparásitas y líquenes, así como evidencias dejadas por hongos, insectos barrenadores y pájaros carpinteros. Finalmente, dado que el tamaño de los árboles puede condicionar la predisposición al decaimiento, la variable diámetro a la altura del pecho (dap) también fue considerada en los análisis (Mencuccini et al., 2005; Bigler, 2016).

\section{MATERIALES Y MÉTODOS}

\section{Área de estudio}

Nothofagus pumilio, regionalmente conocida como lenga, es una especie decidua de amplia distribución en los bosques AndinoPatagónicos extendiéndose por más de $2200 \mathrm{~km}$ en latitud desde los $35^{\circ}$ a $55^{\circ} \mathrm{S}$ y desde el nivel del mar hasta los $2000 \mathrm{~m}$ de elevación (Frangi et al., 2005). Los sitios de estudio se encuentran distribuidos en el norte de la Patagonia entre los $38^{\circ}$ y $42^{\circ} \mathrm{S}$ de latitud y entre $70^{\circ}$ y $72^{\circ} \mathrm{O}$ de longitud (Tabla 1, Fig. 2). En esta región, los veranos son templados y relativamente secos, mientras que los inviernos son fríos y húmedos (Villalba et al., 2003). Las precipitaciones anuales varían entre $4000 \mathrm{~mm}$ en los picos más elevados de la Cordillera de los Andes y de $600 \mathrm{~mm}$ a $800 \mathrm{~mm}$ hacia el este en el ecotono bosque-estepa patagónica (Villalba et al., 2003). Este abrupto cambio en las precipitaciones está inducido por la presencia de la Cordillera que representa una importante barrera para el paso de humedad desde el Océano Pacífico hacia el este.

Las parcelas de muestreo se ubicaron en rodales mayormente monoespecíficos próximos al límite oriental de la distribución de la especie entre 1000 m y 1500 m de altura. Al estar ubicados en el margen seco de su distribución, los bosques de $N$. pumilio son más frecuentemente afectados por estrés hídrico que aquellos ubicados en sectores más húmedos hacia mayores altitudes. Se establecieron 11 parcelas de muestreo en bosques de N. pumilio con decaimiento ubicadas en un transecto latitudinal de más de $500 \mathrm{~km}$ de extensión (Tabla 1), abarcando un total de 457 árboles. Para la selección de las parcelas se tuvieron en cuenta aquellos sectores del bosque que: 1) exhibían posibles indicadores externos de decaimiento, como mortalidad de copa, presencia de ramas apicales muertas, corteza deteriorada, señas y/o presencia de insectos barrenadores y hemiparásitas (Fig. 3) no presentaban indicios de incendios recientes, tala selectiva $\mathrm{y} / \mathrm{o}$ pastoreo intensivo. Asimismo, las parcelas no mostraban evidencias de ataques recientes de insectos defoliadores.
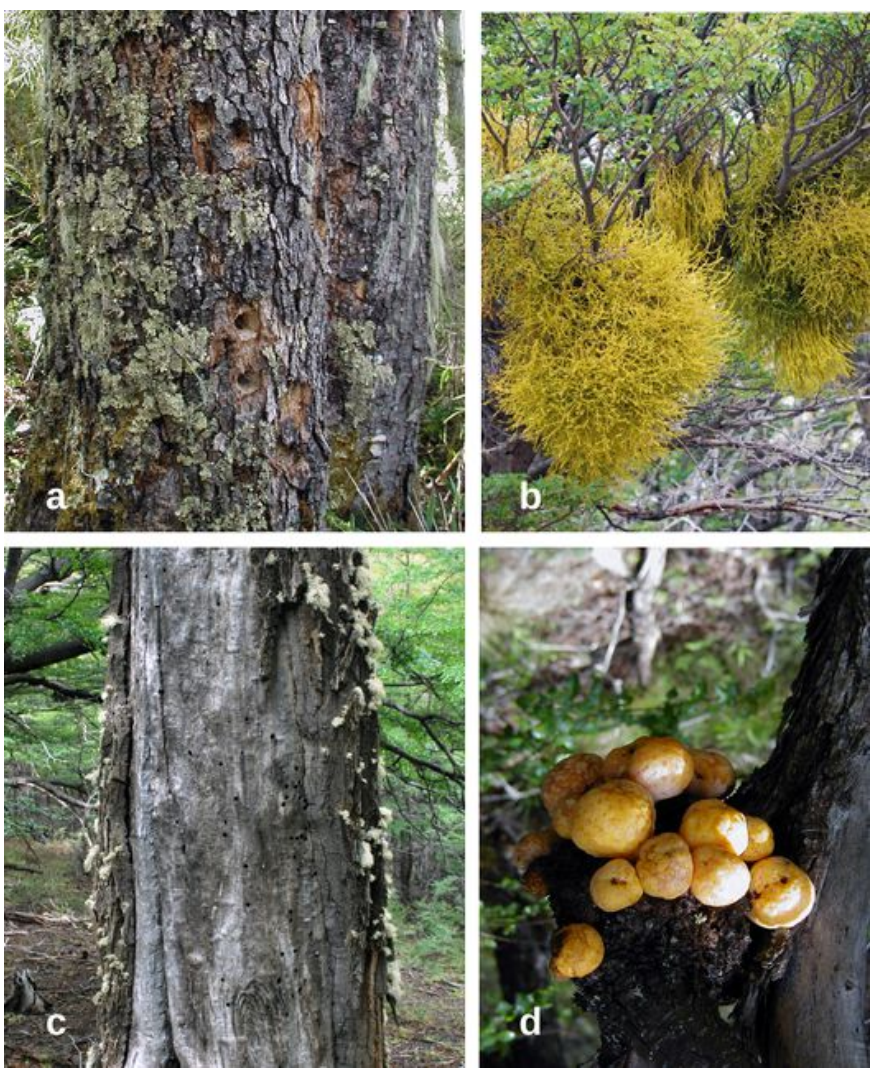

FIGURA 3. Indicadores del decaimiento: a) cavidades producidas por pájaros carpinteros; b) planta hemiparásita (Misodendrum sp.) en la copa de N. pumilio; c) orificios dejados por insectos barrenadores en el fuste de $N$. pumilio con presencia de líquenes en las partes del fuste con corteza; d) nudos o llao llao producidos por hongos parásitos en el fuste de $N$. pumilio.

Con el fin de minimizar los disturbios endógenos y maximizar la señal común del crecimiento entre los individuos de un rodal, al menos 28 árboles vivos fueron incluidos en cada parcela (Cook y Kairiukstis, 2013). Las parcelas fueron rectangulares con su lado menor de por lo menos $20 \mathrm{~m}$ y su lado mayor de $40 \mathrm{~m}$ o más. El tamaño final de cada parcela fue variable en relación con la 
densidad del rodal, pero en todos los casos se incluyeron al menos 40 individuos entre árboles vivos y muertos.

\section{Indicadores externos del decaimiento}

En cada parcela se cuantificaron los indicadores externos que podrían estar asociados al decaimiento en todos los individuos con diámetro a la altura del pecho (dap) mayor a $10 \mathrm{~cm}$. Debido a que la respuesta de los árboles al estrés ambiental varía no solo entre individuos, sino también en función de las condiciones micro-ambientales y la posición en el dosel (Rathgeber, Rossi y Bontemps, 2011), los indicadores externos se evaluaron para la totalidad de los árboles muestreados. De esta forma, todas las situaciones intermedias comprendidas entre árboles sanos contra enfermos, o entre dominantes contra suprimidos, fueron incluidas en el muestreo. En las observaciones de campo, la sanidad de la copa se clasificó en cuatro categorías basadas en el porcentaje de copa muerta (1: $25 \%$ o menos; $2:>25 \%$ y hasta 50\%, 3: > 50\% y hasta 75\%; 4: > 75\%). La sanidad de la corteza fue expresada en porcentaje $(0 \%$ a $100 \%)$ de corteza dañada o muerta en relación al diámetro total del fuste principal a 1,3 m de altura. Por su parte, las (1) galerías de insectos barrenadores (Cerambycidae y Curculionidae; Gentili y Gentili, 1989; McQuillan, 1993; Veblen et al., 1996; Rizzuto, 2009), los (2) orificios de pájaros carpinteros (Campephilus magellanicus), la presencia de (3) plantas hemiparásitas (Misodendrum sp.), (4) hongos Cyttaria sp. (cancrosis) y (5) líquenes (Usnea sp. o Protousnea sp.) fueron consideradas a lo largo de todo el fuste y ramas principales de cada individuo. Estas variables se cuantificaron en escalas de 0 a 3 , siendo 0 : no se observaron signos/presencia ( $\sin$ daños); $1: \leq 25 \%$; ataque/presencia (levemente afectado); 2: $>25 \%$ y $<50 \%$, ataque/presencia (moderadamente afectado); y 3: $>50 \%$, ataque/presencia (muy afectado).

\section{Indicadores externos y crecimiento radial}

La evolución temporal del decaimiento fue inferida a partir de los anillos de crecimiento, considerando a las reducciones sostenidas en el crecimiento como indicadores internos de este proceso. Se compararon los datos de indicadores externos colectados en el trabajo de campo con los patrones de crecimiento de anchos de anillos estandarizados (AA) y de incremento de área basal crudos (IAB) previamente publicados en Rodríguez-Catón et al. (2015).

Con el fin de facilitar el entendimiento del presente trabajo, se explican brevemente en este párrafo algunos métodos y los resultados obtenidos en Rodríguez-Catón et al. (2015). En el mencionado trabajo, las mediciones de AA fueron estandarizadas empleando una línea horizontal correspondiente al valor medio de crecimiento de cada serie para conservar las tendencias de largo plazo que podrían estar asociadas al proceso de decaimiento. Se identificaron las tendencias dominantes de crecimiento de las 11 parcelas utilizando análisis de componentes principales (ACP). El ACP se aplicó a las series de índice de AA e IAB utilizando un período común de $\geq 50$ años que permitió incluir el mayor número posible de árboles por parcela. En la mayoría de las parcelas, los primeros dos o tres componentes principales (CP) representaron al menos $45 \%$ de la varianza total en AA o IAB. Posteriormente, las series individuales de AA e IAB de cada árbol se agruparon de acuerdo con sus correlaciones con los CP ( $\mathrm{r} \geq 0,45)$, y promediaron para desarrollar los patrones dominantes de AA e IAB a nivel de rodal. Los patrones de crecimiento resultantes se extienden desde 1880 hasta el año de muestreo, proporcionando una perspectiva de largo plazo en el crecimiento de los árboles. Cada patrón dominante representa el patrón de crecimiento común de un subconjunto de árboles en cada parcela. Estos patrones dominantes de crecimiento de árboles de las 11 parcelas fueron identificados como patrones de crecimiento local.

En un segundo paso, los patrones de crecimiento locales de las 11 parcelas se correlacionaron entre ellos para identificar patrones comunes a nivel regional. Los patrones de crecimiento local que muestran tendencias similares (correlaciones medias $\mathrm{r} \geq$ $0,3$ y $\mathrm{P}<0,05)$ fueron agrupados posteriormente para obtener los patrones regionales de crecimiento. Con base en el AA y el IAB, dos y tres patrones dominantes de crecimiento regional, respectivamente, fueron identificados (Rodríguez-Catón et al., 2015; Fig. 4). Las cronologías se desarrollaron promediando los índices $\mathrm{AA}$ e IAB crudos de todos los árboles dentro de cada patrón basado en AA e IAB, respectivamente, utilizando el paquete dplR (Bunn, 2008; Bunn et al., 2012; R Core Team, 2017). 


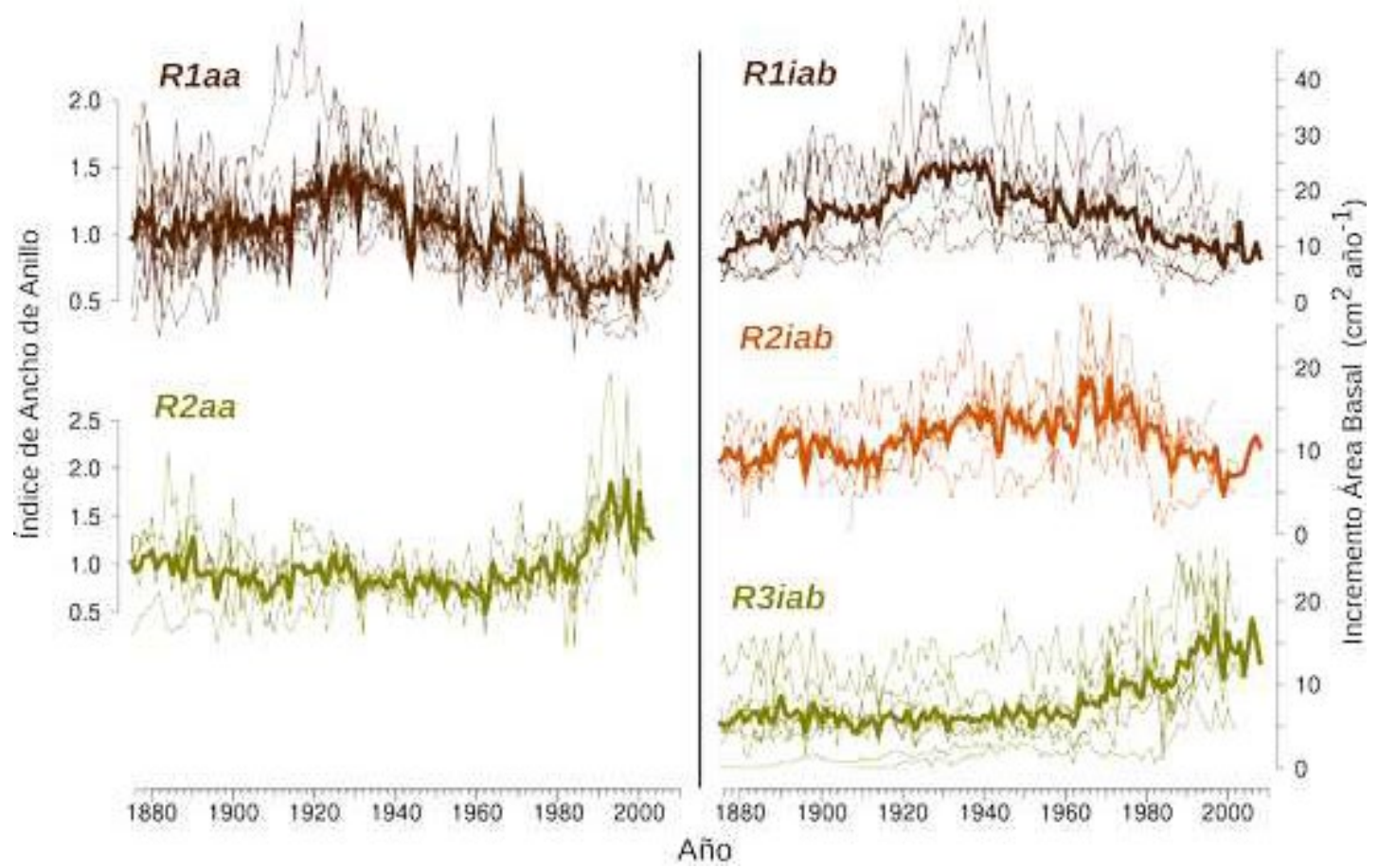

FIGURA 4. Patrones regionales de crecimiento basados en ancho de anillos (AA, izquierda) e incremento en área basal (IAB, derecha).

Las líneas más finas representan los patrones locales de crecimiento que contribuyen a conformar cada patrón regional. Las líneas más gruesas representan los patrones regionales de crecimiento, obtenidos del promedio de los patrones locales más estrechamente relacionados entre sí (Rodríguez-Catón et al., 2015).

En el presente trabajo, se utilizaron los patrones AA siendo renombrados como R1aa y R2aa, mientras que los patrones IAB fueron denominados R1iab, R2iab y R3iab (Fig. 4), respectivamente. La calidad de las cronologías se evaluó mediante el cálculo de las estadísticas Rbar y EPS (Expressed Population Signal) sobre ventanas de 50 años con un desfasaje de 25 años entre segmentos consecutivos. Rbar es la media de todos los coeficientes de correlación resultantes de comparar todos los segmentos de 50 años respectivos entre las series que componen una cronología (Briffa, 1995). La EPS mide la señal total existente en la cronología en relación con una cronología infinitamente replicada (Wigley, Briffa y Jones, 1984). Los valores de EPS cercanos o superiores a 0,85 indican que el número de muestras que integra la cronología en un año determinado retienen una cantidad significativa de la señal teórica presente en una cronología infinitamente replicada.

\section{Pruebas estadísticas}

Con el objeto de evaluar la asociación entre los patrones regionales de crecimiento y cada una de las variables, se utilizó el coeficiente de rangos de Kendall que compara el orden de rangos entre dos variables (Gibbons y Chakraborti, 2003). Este estadístico es no paramétrico y se usó debido a que casi la totalidad de las variables son cualitativas, sin embargo, también asigna rangos a las variables cuantitativas. El coeficiente tau $(\tau)$ de Kendall oscila entre -1 y 1 , es decir entre una relación perfectamente negativa $(\tau=-1)$ hasta una relación positiva perfecta $(\tau=1)$ entre los patrones de crecimiento y las variables (indicadores externos). Asimismo, para discriminar si las muestras (indicadores externos), pertenecen a la misma población (patrones de crecimiento), se procedió a calcular el estadístico de Kruskall-Wallis para los tres patrones regionales IAB. Posteriormente, se calculó el estadístico de Mann-Whitney, cuando la hipótesis nula del test de Kruskall-Wallis fue rechazada (se rechaza que las muestras pertenecen a la misma población).

En el caso de los dos patrones regionales AA, se realizó solamente la prueba de Mann-Whitney. Esta prueba, también conocida como test de Wilcoxon, es una alternativa no paramétrica de la prueba t de Student para contrastar si dos o más muestras provienen de una misma población, basándose en la 
suma de rangos asignados a las observaciones (Ríus Díaz, Barón Lopez, Sánchez Font y Parras Guijosa, 2010). Para hacer más riguroso el análisis, se utilizó el ajuste Bonferroni con un valor de significancia 0,05 dividido por la cantidad de pruebas que se realizaron, normalmente dos o tres, según el número de patrones de crecimiento. La clasificación de los árboles estuvo basada en las categorías correspondientes a cada patrón regional de crecimiento tanto de IAB como AA. Los análisis fueron ejecutados con el software libre R (R Core Team, 2017), utilizando las funciones (paquetes): aov (stats), shapiro.test (stats), Kendall (Kendall); kruskal.test (stats) y wilcox_test (coin). Se emplearon gráficos de caja y bigotes para caracterizar los grupos de árboles con base en cada patrón de crecimiento.

\section{Resultados}

\section{Cronologías de los patrones regionales de crecimiento}

En un estudio previo, Rodríguez-Catón et al. (2015) obtuvieron dos y tres patrones regionales de crecimiento, basados en AA e IAB, respectivamente (Fig. 4). Las cronologías desarrolladas con base en estos patrones regionales de crecimiento están compuestas entre 54 y 263 árboles para los patrones basados en AA y 90, 96 y 108 árboles para los patrones elaborados a partir del IAB (Tabla 2). Las estadísticas de las cronologías indican que el valor de correlación medio entre las series es de entre 0,209 y 0,44 (Rbar). El EPS indica que estas cronologías están suficientemente replicadas para el periodo 1895-2004.

\section{Relación entre el crecimiento y los indicadores externos del decaimiento}

Las relaciones entre los patrones regionales de crecimiento basados en el IAB con los indicadores externos mostraron significativas para las variables: mortalidad de copa, porcentaje de corteza muerta, insectos barrenadores, pájaros carpinteros y dap (Tabla 3). La relación entre cada una de estas variables y el crecimiento fue inversa, es decir, a menor crecimiento durante las últimas décadas (R1iab y R2iab), mayor porcentaje de mortalidad de copa, mayor porcentaje de corteza muerta, mayor incidencia de insectos barrenadores, mayor cantidad de orificios de pájaros carpinteros y mayor dap. Por otro lado, la cantidad de líquenes mostró una relación directa, que indica que a menor crecimiento expresado en IAB, menor cantidad de líquenes.
Las mismas variables mencionadas en el párrafo anterior, sumadas a la cantidad de plantas hemiparásitas (misodendron), presentaron valores elevados de $\mathrm{chi}^{2}$, y altas significancias estadísticas en la prueba de Kruskall-Wallis (Tabla 3). El resultado del test de Kruskall-Wallis para el dap coincide con los resultados del ANOVA realizado para esta variable (resultados no mostrados). El test de Mann-Whitney reveló que los individuos representados por el patrón regional R1iab (tendencia negativa en el crecimiento desde la década de 1940) tuvieron significativamente mayor mortalidad de copa, mayor porcentaje de corteza muerta y mayor incidencia de insectos barrenadores que aquellos provenientes de patrones con tendencias positivas (R3iab) y/o negativas (R2iab) que se iniciaron más recientemente (Fig. 5). A su vez, los individuos representados por el patrón con tendencia negativa de IAB en las últimas tres décadas (R2iab) tuvieron mayor mortalidad de copa, mayor porcentaje de corteza muerta y mayor incidencia de insectos barrenadores que aquellos representados por el patrón de crecimiento con tendencia positiva en IAB (R3iab).

Del total de 186 árboles con tendencias negativas en el crecimiento, 16 árboles (9\%) y 31 árboles (17\%) pertenecientes a los patrones R1iab y R2iab, respectivamente, presentaron condiciones sanitarias buenas: $<25 \%$ de copa muerta, sin corteza dañada y sin orificios producto de la actividad de insectos barrenadores. En su mayoría, los árboles con tendencias positivas de crecimiento (R3iab) presentaron $<25 \%$ de copa muerta, prácticamente sin daños en la corteza, ni orificios de insectos barrenadores (Fig. 5). Solo se registró un porcentaje menor (12\%) de árboles con tendencias positivas en el crecimiento con más de $50 \%$ de la copa muerta y un $41 \%$ de la corteza afectada, en promedio. La incidencia de pájaros carpinteros aumentó significativamente en aquellos árboles con tendencias negativas de más larga data (R1bai), en comparación con aquellos de tendencia negativa más reciente (R2bai) y tendencia positiva (R3bai; Fig. 5). Por el contrario, las variaciones en la abundancia de Misodendrum sp., Cyttaria sp. y líquenes no están significativamente relacionadas con los distintos patrones IAB. Si bien las variables Misodendrum sp. y líquenes también muestran diferencias significativas (patrón R2iab vs. R1iab y R3iab, y patrón R2iab vs. R3iab, respectivamente), patrones con tendencias de crecimiento marcadamente opuestas como el R1iab y el R3iab no presentan diferencias entre estas variables (Fig. 5). 
TABLA 2. Estadísticas de las cronologías de los patrones regionales de crecimiento.

\begin{tabular}{lccccc}
\hline & \multicolumn{2}{c}{ Ancho de anillo (AA) } & \multicolumn{2}{c}{ Incremento de área basal (IAB) } \\
\hline Patrón Regional & Rlaa & R2aa & Rliab & R2iab & R3iab \\
\hline No. de árboles & 263 & 54 & 90 & 96 & 108 \\
Periodo & $1677-2008$ & $1668-2004$ & $1716-2008$ & $1677-2008$ & $1668-2008$ \\
EPS $>0,85$ & $1785-2008$ & $1885-2004$ & $1895-2008$ & $1830-2008$ & $1895-2008$ \\
\hline Rbar medio & 0,303 & 0,209 & 0,296 & 0,232 & 0,440 \\
\hline
\end{tabular}

TABLA 3. Coeficiente de rangos de Kendall $(\tau)$ para los patrones de crecimiento IAB y AA contra los indicadores externos. También se muestran los valores $\mathrm{Chi}^{2}$ de la prueba de Kruskall-Wallis para los patrones IAB y los indicadores externos.

\begin{tabular}{llll}
\hline & $\tau$ IAB & $\tau$ AA & Kruskall-Wallis IAB (Chi $)$ \\
\hline Mortalidad de copa & $-0,428^{* * *}$ & $-0,108^{*}$ & $71,014^{* * *}$ \\
Corteza muerta & $-0,258^{* * *}$ & $-0,00495$ & $26,068^{* * *}$ \\
Barrenadores & $-0,312^{* * *}$ & $-0,0492$ & $36,682^{* * *}$ \\
Carpinteros & $-0,265^{* * *}$ & $-0,145^{*}$ & $25,422^{* * *}$ \\
Misodendrum sp. & $0,04^{* *}$ & $-0,0501$ & $16,603^{* * *}$ \\
Cyttaria sp. & 0,0458 & $-0,0256$ & 1,5084 \\
Líquenes & $0,114^{*}$ & 0,0644 & $10,469^{* *}$ \\
\hline dap & $-0,24^{* * *}$ & 0,0314 & $31,15^{* * *}$ \\
\hline
\end{tabular}

Los asteriscos indican los valores de $\mathrm{p:}{ }^{*}<0,05 ;{ }^{* *}<0,01 ;{ }^{* * *}<0,001$.
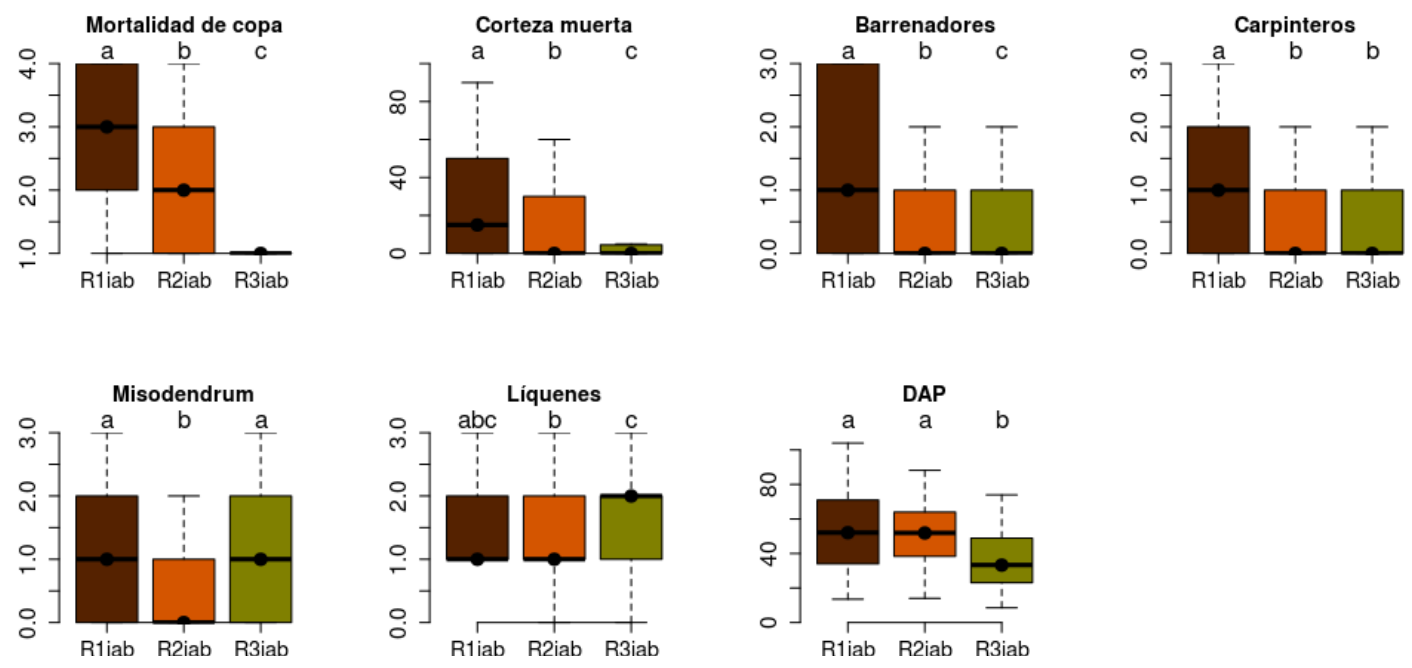

FIGURA 5. Relación entre los patrones regionales de crecimiento expresado en IAB (R1iab, R2iab y R3iab) y las variables analizadas: mortalidad de copa, corteza muerta, barrenadores, pájaros carpinteros, Misodendrum sp., líquenes y dap. Diferentes letras indican diferencias significativas entre los patrones, según los resultados de las pruebas no paramétricas de Mann-Whitney. 
En cuanto a los patrones de crecimiento expresados en ancho de anillos (AA), se detectaron relaciones significativas entre árboles del patrón R1aa y R2aa para las variables porcentaje de mortalidad de copa y cavidades de pájaros carpinteros. Las relaciones fueron inversas, indicando que, al aumentar la mortalidad de copa y las cavidades producidas por carpinteros, disminuye el crecimiento (Tabla 3). Estas mismas variables fueron significativas para la prueba de Mann-Whitney (Fig. 6). El resto de las variables externas consideradas, no registraron diferencias significativas empleando anchos de anillos.

\section{DISCUSIÓN}

Entre los indicadores externos analizados, el grado de afectación de la copa fue la variable que mostró relaciones más estrechas con los patrones de crecimiento de N. pumilio. Este indicador externo se asocia significativamente con todos los patrones regionales de crecimiento, tanto aquellos basados en el AA como en el IAB. Árboles con porcentajes elevados de mortalidad de copa presentaron, en su mayoría, reducciones marcadas en sus tasas de crecimiento, mientras que los árboles de copas sanas muestran principalmente tendencias positivas en el crecimiento. Sin embargo, 47 de un total de 186 árboles sin mortalidad manifiesta de copa registraron tendencias negativas (declinación) en el crecimiento durante las últimas décadas. Esto indica que en aproximadamente un $25 \%$ de los individuos la manifestación externa del decaimiento asociada con la defoliación y muerte parcial de la copa ocurre posteriormente al inicio de la declinación registrada en el ancho de los anillos de crecimiento y, por lo tanto, la mortalidad de copa como único atributo podría estar subestimando el nivel real de decaimiento del rodal (Duchesne, Ouimet y Morneau, 2003).

Por otro lado, la estimación a campo del porcentaje de la mortalidad de copa puede introducir algunos errores de apreciación que finalmente contribuyen a las diferencias observadas. Precisar si la mortalidad de copa de un individuo alcanza $24 \%$ o $26 \%$ del total de la copa no es una tarea sencilla y fácil de establecer en el campo. Sin embargo, esta pequeña diferencia de solo $2 \%$ determina que un individuo sea categorizado como 4 o 3 en relación con su mortalidad de copa. En contraposición, también se observó que 12\% de árboles con tendencias positivas de crecimiento presentaban más de $50 \%$ de la copa muerta y, en promedio, $41 \%$ de la corteza afectada. Se ha notado, que en aquellos árboles donde se producen daños parciales de la corteza alrededor del tronco principal, el crecimiento se concentra en el sector vivo del árbol. Por lo tanto, en muestras de barreno obtenidas en los sectores vivos del tronco en individuos con mortalidad parcial de la corteza, el incremento radial refleja la concentración del crecimiento en el sector vivo del fuste originando una sobreestimación del crecimiento en individuos con altos porcentajes de mortalidad de la corteza.
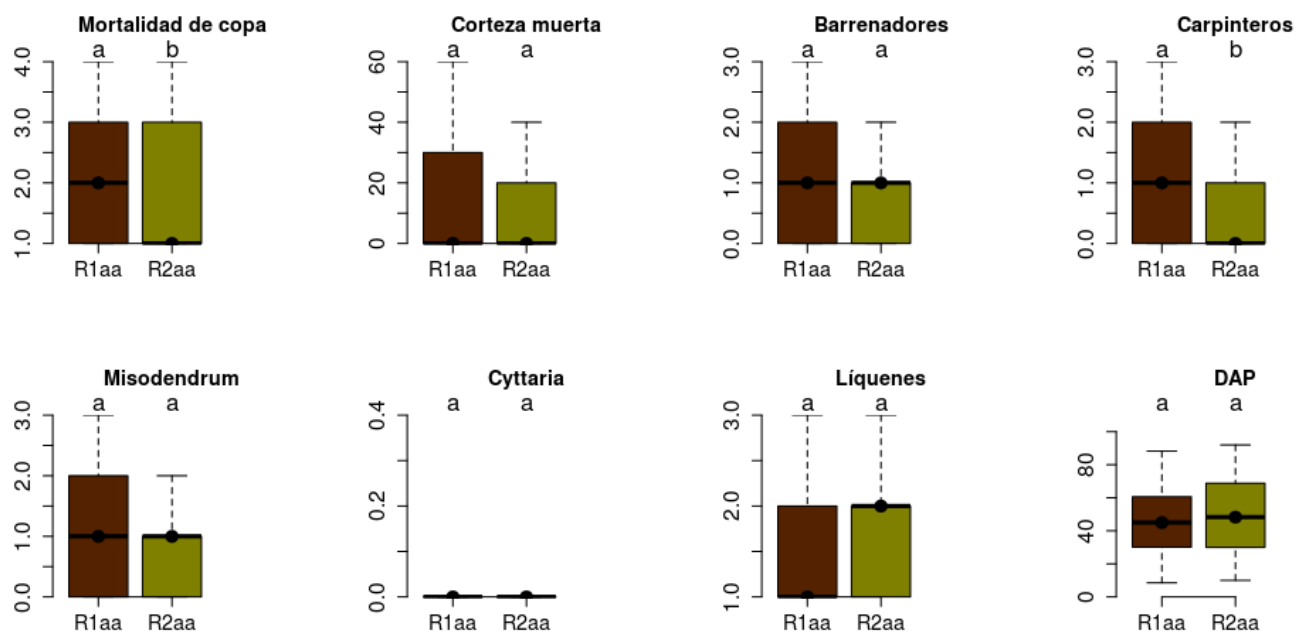

FIGURA 6. Relación entre los patrones regionales de crecimiento expresado en AA (R1aa y R2aa) y las variables analizadas: mortalidad de copa, corteza muerta, barrenadores, pájaros carpinteros, Misodendrum sp., Cyttaria sp., líquenes y dap.

Diferentes letras indican diferencias significativas entre los patrones, según los resultados de las pruebas no paramétricas de Mann-Whitney. 
Efectivamente, en este estudio se notó que los pocos individuos que muestran este patrón contrapuesto $(>50 \%$ de mortalidad de copa y alto crecimiento radial) presentan un elevado porcentaje de la corteza muerta (resultados no mostrados). Diferencias en el ritmo de crecimiento entre sectores del fuste han sido también observadas en ejemplares de $N$. pumilio cuyos troncos presentaban parte de su corteza muerta por el efecto de avalanchas de nieve (Casteller, Villalba y Mayer, 2009). En el presente estudio, que cubre una amplia franja latitudinal, es muy probable que condiciones de micrositio o historias particulares de disturbios también contribuyan a originar tendencias en el crecimiento o indicadores externos de decaimiento no totalmente consistentes con las registradas para la mayoría de árboles a escala regional.

El porcentaje de corteza dañada, la presencia de orificios causados por insectos barrenadores y las cavidades producidas por pájaros carpinteros, a nivel regional, también tuvieron mayor incidencia en árboles con tendencias negativas en el crecimiento. Suarez et al., (2004) observaron que los individuos muertos de $N$. dombeyi a causa de la sequía ocurrida, durante 1998-1999 en el Parque Nacional Nahuel Huapi tenían, previo a la sequía, una mayor incidencia de insectos barrenadores que los árboles sanos. Estos autores no encontraron diferencia en la incidencia de pájaros carpinteros (Campephilus magellanicus) comparando rodales sanos y afectados por sequía. Sin embargo, estudios posteriores en los bosques de N. pumilio del norte de la Patagonia mostraron que el carpintero Campephilus magellanicus prefiere construir sus cavidades en árboles con crecimiento radial reducido y grados avanzados de mortalidad de copa (Ojeda et al., 2007). Además, los árboles con cavidades comenzaron sincrónicamente sus tendencias negativas en el crecimiento luego de las sequías de 1943-1944 y 1956-1957 y defoliaciones masivas producidas por Ormiscodes sp. durante 1985-1986.

En el presente estudio, las cavidades de pájaros carpinteros también mostraron diferencias entre árboles con tendencias negativas de más largo plazo y tendencias positivas tanto en AA como en IAB, aunque no se observaron diferencias significativas entre los árboles con tendencias negativas más recientes $\mathrm{y}$ aquellos con tendencias positivas en IAB (R2iab vs. R3iab). Estos resultados sugirieren que la afectación de tronco con orificios dejados por pájaros carpinteros ocurre luego de varios años de haberse iniciado el decaimiento. Debido a que Campephilus magellanicus se alimenta principalmente de insectos barrenadores (Chazarreta et al., 2012; Ojeda, 2004), la presencia de esta ave puede ser consecuencia de la abundancia de barrenadores.

Estudios previos muestran que el crecimiento radial en los bosques de $N$. pumilio cercanos al límite con la estepa (mayor aridez) está modulado principalmente por la disponibilidad hídrica (Rodríguez-Catón et al., 2016). Estos autores propusieron una combinación de eventos climáticos que predisponen a los individuos de N. pumilio al decaimiento. Periodos húmedos prolongados (alrededor de una década) que favorecieron el desarrollo de copas frondosas y acelerados ritmos de crecimiento, fueron interrumpidos por sequías extremas (Rodríguez-Catón et al., 2016). Según Manion (1981, 2003), estos individuos afectados en sus sistemas de conducción incrementan su susceptibilidad a posteriores sequías o ataques de plagas o enfermedades. Integrando los estudios anteriores con el presente trabajo se propone que el decaimiento de $N$. pumilio se manifiesta a través de la disminución sostenida del crecimiento, altos porcentajes de mortalidad de copa y de corteza, condiciones que favorecen la actividad de insectos barrenadores. Así, los árboles que experimentan un proceso de disminución del crecimiento causado por sequías son más susceptibles al ataque de insectos que se ven favorecidos por el debilitamiento de los individuos. De esta manera, los individuos debilitados que no logran recuperarse son más propensos a la muerte debida al agotamiento de reservas, al ataque o intensificación de agentes bióticos y la ocurrencia de nuevos eventos de sequías. La evaluación en campo de los indicadores externos del decaimiento junto con la determinación dendrocronológica del crecimiento, permiten describir el estado sanitario general de un árbol o un rodal y su susceptibilidad ante nuevas situaciones de estrés, ampliando asimismo el conocimiento relacionado al complejo proceso de decaimiento forestal.

Por otro lado, la presencia de hemiparásitas del género Misodendrum, hongos del género Cyttaria, y líquenes del género Usnea y Protousnea no mostraron relaciones con el decaimiento evaluado tanto a partir de indicadores internos (crecimiento) como externos (mortalidad de copa) del decaimiento. En el caso particular de la incidencia de hemiparásitas, los resultados de este estudio son contrastantes con los de Henríquez-Velásquez et al. 
(2012), quienes observaron que la presencia de Misodendrum punctulatum en ejemplares de N. pumilio en la Patagonia Sur $\left(52^{\circ} \mathrm{S}\right)$ es mayor en árboles con reducción en el crecimiento radial y menor sanidad de copa. Para el norte de la Patagonia, Suarez et al. (2004) también analizaron las diferencias entre rodales sanos y afectados de $N$. dombeyi luego de la sequía de 1998-1999, en cuanto a la incidencia de Misodendrum sp. y Cyttaria sp. sin encontrar diferencias entre los rodales. Sin embargo, esta falta de concordancia en los resultados puede deberse a diferencias en los métodos de evaluación de la presencia de Misodendrum sp. Por lo tanto, estudios más detallados son necesarios para evaluar más precisamente la preferencia de estas especies por árboles sanos o afectados por decaimiento.

En general, los indicadores externos estuvieron más fuertemente relacionadas con patrones dominantes del crecimiento con tendencias negativas, evaluadas tanto a través del ancho de los anillos (R1aa) o los incrementos en área basal (R1iab y R2iab). Sin embargo, el patrón de crecimiento expresado en IAB que posee tendencia negativa de más largo plazo (R1iab) se asocia con valores más altos de mortalidad de copa, corteza afectada, orificios producidos por insectos barrenadores y cavidades producidas por pájaros carpinteros. Por lo tanto, las relaciones entre los indicadores externos y los patrones dominantes de crecimiento son más consistentes cuando las tendencias de crecimiento se expresan en IAB. Las diferencias entre el empleo del AA o IAB están asociadas a las diferencias en las tendencias del crecimiento de los árboles registradas entre ambas variables. En general, el AA disminuye geométricamente a medida que se incrementa el diámetro de los árboles (tendencias negativas; Fritts, 1976). Por el contrario, el área basal que contabiliza el total del crecimiento diamétrico puede mantenerse estable o en aumento a medida que se incrementa el diámetro de un árbol.

Estas diferencias en la forma de evaluar las variaciones interanuales en el crecimiento de los árboles influencian largamente las diferencias observadas. Debido al mencionado efecto geométrico de reducción en el espesor del ancho de anillos impuesto por el crecimiento radial, los trabajos que intenten comparar las tendencias de largo plazo en el crecimiento con otros parámetros de la dinámica forestal deberían tener en cuenta el efecto de esta reducción geométrica en el crecimiento diametral cuando se emplean los valores brutos del ancho de los anillos. Por ello, la mayoría de los estudios de este tipo prefieren usar el IAB frente al ancho de anillos para estudiar el vigor y estado sanitario de los bosques (Duchesne et al., 2003; Rodríguez-Catón et al., 2016). Sin embargo, son escasos los estudios que comparan ambas estimaciones del crecimiento con respecto a indicadores externos del decaimiento.

\section{CONCLUSIONES}

Debido al alto porcentaje de árboles con copas parcialmente muertas y patrones de declinación o reducción en el crecimiento, los resultados de este trabajo indican, de acuerdo con los criterios establecidos por Dobbertin (2005), una escasa vitalidad en los bosques de $N$. pumilio a lo largo de su distribución oriental en el norte de la Patagonia argentina. Los árboles con tendencias positivas en el crecimiento podrían equiparar las pérdidas producidas por el decaimiento, siempre y cuando no sean afectados en un futuro próximo también por este proceso. Ello indica que es importante realizar, junto con el análisis dendrocronológico, un seguimiento de estos bosques a través de inventarios forestales, como también compararlos con masas forestales que no manifiesten decaimiento. Esto contribuirá a ampliar la información sobre la evolución de la vitalidad y el decaimiento de los bosques de $N$. pumilio cercanos al límite oriental de su distribución.

Utilizando la dendrocronología y una estrategia sencilla de observación en campo, los resultados de este estudio muestran que las tasas de crecimiento observados, junto con las tendencias de crecimiento y el porcentaje de mortalidad de copa, pueden ser de gran utilidad para evaluar el grado de decaimiento de las especies leñosas. Los estudios que incluyan estas tres variables podrían mejorar los modelos de predicción de mortalidad de árboles (Gillner, Rüger, Roloff y Berger, 2013). Otros indicadores, relacionados con el crecimiento y de rápida cuantificación en las tareas de campo como sanidad de corteza, incidencia de insectos barrenadores y pájaros carpinteros, podrían ser utilizados en bosques de $N$. pumilio para identificar rodales o individuos con decaimiento o ser empleados para desarrollar índices de decaimiento en distintos parches forestales de la Patagonia. 


\section{RECONOCIMIENTOS}

Los autores agradecen el apoyo del Consejo Nacional de Investigaciones Científicas y Técnicas (Conicet; PIP 1122011010- 0809), Argentina. También agradecen por el trabajo de S. Calí, S. Delgado, M. Masiokas, A. Ripalta, A. Srur y R. Urrutia, durante las tareas de campo. La contribución de tres revisores anónimos fue a su vez muy valiosa para este artículo.

\section{REFERENCIAS}

Allen, C. D., Macalady, A. K., Chenchouni, H., Bachelet, D., McDowell, N. G., Vennetier, M., \& Cobb, N. (2010). A global overview of drought and heat-induced tree mortality reveals emerging climate change risks for forests. Forest Ecology and Management, 259(4), 660684. doi: 10.1016/j.foreco.2009.09.001

Amoroso, M. M., \& Larson, B. C. (2010). Stand development patterns as a consequence of the mortality in Austrocedrus chilensis forests. Forest Ecology and Management, 259(10), 1981-1992. doi: 10.1016/j.foreco.2010.02.009

Anderegg, W. R. L., Kane, J. M., \& Anderegg, L. D. L. (2013). Consequences of widespread tree mortality triggered by drought and temperature stress. Nature Climate Change, 3(1), 30-36. doi: 10.1038/nclimate1635

Attis Beltrán, H., Martínez Pastur, G., Ivancich, H., Lencinas, M. V, \& Chauchard, L. M. (2013). Tree health influences diameter growth along site quality, crown class and age gradients in Nothofagus forests of southern Patagonia. Journal of Forest Science, 59(8), 328336.

Bigler, C. (2016). Trade-Offs between Growth Rate, Tree Size and Lifespan of Mountain Pine (Pinus montana) in the Swiss National Park. PloS One, 11(3), 1-18. doi: 10.5061/dryad.d2680

Bowers, J. E., \& Turner, R. M. (2001). Dieback and Episodic Mortality of Cercidium microphyllum (foothill paloverde), a dominant Sonoran Desert Tree. Journal of the Torrey Botanical Society, 128(2), 128-140. doi: 10.2307/3088735.

Briffa, K. R. (1995). Interpreting high-resolution proxy climate data- the example of dendroclimatology. En H. Von Storch \& A. Navarra (Eds.), Analysis of climate variability, applications of statistical techniques (pp. 77-94). Springer, Berlin.

Brodo, I. (1973). Substrate Ecology. En V. Ahmadjian \& M. E. Hale (Eds.), The Lichens (pp. 401-441). Nueva York, Estados Unidos: Academic Press.

Bunn, A. G. (2008). A dendrochronology program library in R (dplR). Dendrochronologia, 26(2), 115-24. doi: 10.1016/j.dendro. 2008.01.002.

Bunn, A. G., Korpela, M., Biondi, F., Merian, P., Qeadan, F., \& Zang, C. (2012). dplR: Dendrochronology Program Library in R. R package version 1.5.5. Recuperado de http://cran.rproject.org $/$ package $=\mathrm{dplR}$.

Cailleret, M., Nourtier, M., Amm, A., Durand-Gillmann, M., \& Davi, H. (2013). Drought-induced decline and mortality of silver fir differ among three sites in Southern France. Annals of Forest Science, 71(6), 643-657. doi: 10.1007/s13595-013-0265-0.

Calí, S. (1996). Austrocedrus chilensis: estudio de los anillos de crecimiento y su relación con la dinámica del "mal del cipres" en el parque nacional Nabuel Huapi (tesis de pregrado). Universidad Nacional del Comahue, Bariloche, Argentina.

Calvelo, S., Stocker-Wörgöter, E., Liberatore, S., \& Elix, J. (2005). Protousnea (Parmeliaceae: Ascomycota), a genus endemic to Southern South America. Bryologist, 108, 1-15. doi: 10.1639/00072745(2005)108[1:PPAAGE]2.0.CO;2.

Casteller, A., Villalba, R., \& Mayer, A. (2009). Reconstrucción espacial y temporal de la ocurrencia de avalanchas de nieve en los Andes patagónicos utilizando técnicas dendrocronológicas. Revista Chilena de Historia Natural, 82, 245-264. doi: 10.4067/S0716078X2009000200007.

Chazarreta, L., Ojeda, V., \& Lammertink, M. (2012). Morphological and foraging behavioral differences between sexes of the magellanic woodpecker (Campephilus magellanicus). Ornitologia Neotropical, 23, 529-544.

Ciesla, W. M., \& Donaubauer, E. (1994). Decline and dieback of trees and forests. A global overview. Rome: Food \& Agriculture Org.

Cook, E., \& Kairiukstis, L. A. (2013). Methods of dendrochronology: applications in the environmental sciences. Estados Unidos: Springer Science \& Business Media.

Dobbertin, M. (2005). Tree growth as indicator of tree vitality and of tree reaction to environmental stress: a review. European Journal of Forest Research, 124(4), 319-333. doi: 10.1007/s10342-005-0085-3.

Duchesne, L., Ouimet, R., \& Morneau, C. (2003). Assessment of sugar maple health based on basal area growth pattern. Canadian Journal of Forest Research, 33(1996), 2074-2080. doi: 10.1139/X03-141.

Frangi, J. L., Barrera, M. D., Puigdefábregas, J., Yapura, P. F., Arambarri, A. M., \& Richter, L. L. (2005). Ecología de los bosques de tierra del fuego. En M. F. Arturi, J. L. Frangi, \& J. F. Goya (Eds.), Ecologia y manejo de los bosques de Argentina. Argentina: Editorial de la Universidad Nacional de La Plata (EDULP).

Franklin, J. F., Shugart, H. H., \& Harmon, M. E. (1987). Tree death as an ecological process. The causes, consequences, and variability of tree mortality. BioScience, 37(8), 550-556.

Fritts, H. (1976). Tree Rings and Climate. Londres, Inglaterra: Academic Press.

Gentili, M., \& Gentili, R. (1989). Lista comentada de los insectos asociados a las especies sudamericanas del género Nothofagus. En I. Gamundi (Ed.), Simposio sobre Nothofagus. 
Gibbons, J., \& Chakraborti, S. (2003). Nonparametric Statistical Inference (pp. 1-645).

Gillner, S., Rüger, N., Roloff, A., \& Berger, U. (2013). Low relative growth rates predict future mortality of common beech (Fagus sylvatica L.). Forest Ecology and Management, 302, 372-378. doi: 10.1016/j.foreco.2013.03.032.

Henríquez-Velásquez, C., Henríquez, J., \& Aravena, J. (2012). Damage caused by mistletoe Misodendrum punctulatum Banks Ex Dc. on architecture and radial growth of Nothofagus pumilio (Poepp. et Endl.) Krasser forests of southern Chile. Austral Ecology, 37(7), 816-824. doi: 10.1111/j.1442-9993.2011.02342.x.

Hogg, E. H. (Ted), Brandt, J. P., \& Michaelian, M. (2008). Impacts of a regional drought on the productivity, dieback, and biomass of western Canadian aspen forests. Canadian Journal of Forest Research, 38(6), 1373-1384. doi: 10.1139/X08-001.

Ireland, K. B., Moore, M. M., Fulé, P. Z., Zegler, T. J., \& Keane, R. E. (2014). Slow lifelong growth predisposes Populus tremuloides trees to mortality. Oecologia, 175(3), 847-59. doi: 10.1007/s00442014-2951-5.

Kolb, T. E., \& Mccormick, L. H. (1993). Etiology of sugar maple decline in 4 Pennsylvania stands. Canadian Journal of Forest Research, 23(11), 2395-2402. doi: 10.1139/x93-296

Manion, P. D. (1981). Tree disease concepts. Nueva Jersey, Estados Unidos: Prentice Hall.

Manion, P. D. (2003). Evolution of Concepts in Forest Pathology. Phytopathology, 93, 1052-1055. doi: 10.1094/PHYTO. 2003.93.8.1052.

McDowell, N. G., Ryan, M. G., Zeppel, M. J. B., \& Tissue, D. T. (2013). Improving our knowledge of drought-induced forest mortality through experiments, observations, and modeling. New Phytologist, 200, 289-293. doi: 10.1111/nph.12502.

McQuillan, P. B. (1993). Nothofagus (Fagaceae) and its invertebrate faunaan overview and preliminary synthesis. Biological Journal of the Linnean Societty, 49(September).

Mencuccini, M., Martínez-Vilalta, J., Vanderklein, D., Hamid, H. a, Korakaki, E., Lee, S., \&Michiels, B. (2005). Size-mediated ageing reduces vigour in trees. Ecology Letters, 8(11), 1183-90. doi: 10.1111/j.1461-0248.2005.00819.x.

Montoya Oliver, M. J. (1995). Efecto del cambio climático sobre los ecosistemas forestales españoles. Cuadernos de La Sociedad Española de Ciencias Forestales, 2(65), 65-76.

Mueller-Dombois, D. (1992). A natural dieback theory, cohort senescence as an alternative to the decline desease theory. En P. D. Manion, \& D. Lachance (Eds.). Forest decline concepts (pp. 2637). APS Press.

Mundo, I. A., El Mujtar, V. A., Perdomo, M. H., Gallo, L. A., Villalba, R., \& Barrera, M. D. (2010). Austrocedrus chilensis growth decline in relation to drought events in northern Patagonia, Argentina. Trees, 24(3), 561-570. doi: 10.1007/s00468-010-0427-8.

Ohno, Y., Umeki, K., Watanabe, I., Takiya, M., Terazawa, K., Yasaka, M., \& Matsuki, S. (2008). Basal area growth and mortality of Betula maximowicziana affected by crown dieback in a secondary forest in Hokkaido, northern Japan. Journal of Forest Research, 14(1), 37-43. doi: 10.1007/s10310-008-0103.

Ojeda, V. S. (2004). Breeding biology and social behaviour of Magellanic Woodpeckers (Campephilus magellanicus) in Argentine Patagonia. European Journal of Wildlife Research, 50, 18-24. doi: 10.1007/s10344-003-0029-5.

Ojeda, V. S., Suarez, M. L., \& Kitzberger, T. (2007). Crown dieback events as key processes creating cavity habitat for magellanic woodpeckers. Austral Ecology, 436-445. doi: 10.1111/j.14429993.2007.01705.x.

R Core Team. (2017). R: A Language and Environment for Statistical Computing. R Foundation for Statistical Computing. Vienna, Austria: R Foundation for Statistical Computing.

Rathgeber, C. B. K., Rossi, S., \& Bontemps, J.-D. (2011). Cambial activity related to tree size in a mature silver-fir plantation. Annals of Botany, 108(3), 429-38. doi: 10.1093/aob/mcr168.

Rice, K. J., Matzner, S. L., Byer, W., \& Brown, J. R. (2004). Patterns of tree dieback in Queensland, Australia: the importance of drought stress and the role of resistance to cavitation. Oecologia, 139(2), 190-8. doi: 10.1007/s00442-004-1503-9.

Ríus Díaz, F., Barón Lopez, F., Sánchez Font, E., \& Parras Guijosa, L. (2010). Bioestadística: Métodos y aplicaciones (p. 322). Recuperado de http://www.citeulike.org/user/Bibliosaude/article/8420366.

Rizzuto, S. (2009). Observations on the larval biology in the genus Calydon (Coleoptera: Cerambycidae) with new records in Patagonia, Argentina. Revista de La Sociedad Entomologica Argentina, 68(3-4), 391-396.

Rodríguez-Catón, M., Villalba, R., Morales, M., \& Srur, A. (2016). Influence of droughts on Nothofagus pumilio forest decline across northern Patagonia, Argentina. Ecosphere, 7(July), 1-17. doi: 10.1002/ecs2.1390.

Rodríguez-Catón, M., Villalba, R., Srur, A. M., \& Luckman, B. (2015). Long-term trends in radial growth associated with Nothofagus pumilio forest decline in Patagonia: Integrating local- into regionalscale patterns. Forest Ecology and Management, 339, 44-56. doi: 10.1016/j.foreco.2014.12.004.

Steinkamp, J., \& Hickler, T. (2015). Is drought-induced forest dieback globally increasing? Journal of Ecology, 103(1), 31-43. doi: 10.1111/1365-2745.12335.

Suarez, M. L., Ghermandi, L., \& Kitzberger, T. (2004). Factors predisposing episodic drought-induced tree mortality in Nothofagus - site, climatic sensitivity and growth trends. Journal of Ecology, 92, 954-966. doi: 10.1111/j.1365-2745.2004.00941.x. 
Veblen, T., Donoso, C, Kitzberger, T., \& Rebertus, A. J. (1996). Ecology of southern chilean and argentinean Nothofagus forests. En Veblen, T., Hill, R. S. y Read, J. (Eds.), The Ecology and Biogeography of Nothofagus forests. Estados Unidos: Yale University Press.

Vila-Cabrera, A., Martínez-Vilalta, J., Galiano, L., \& Retana, J. (2013). patterns of forest decline and regeneration across Scots Pine populations. Ecosystems, 16, 323-335. doi: 10.1007/s10021-0129615-2.

Villalba, R., Lara, A., Masiokas, M., Delgado, S., Aravena, J. C., Roig, F. A., \& Ripalta, A. (2003). Large-scale temperature changes across the southern andes: 20th-century variations in the context of the past 400 years. Climatic Change, 59, 177-232. doi: 10.1023/A:1024452701153.

Villalba, R., \& Veblen, T. (1998). Influences of large-scale climatic variability on episodic tree mortality in northern Patagonia. Ecology, 79(8), 2624-2640. doi: 10.1890/00129658(1998)079[2624:IOLSCV]2.0.CO;2.

Wigley, T. M. L., Briffa, K. R., \& Jones, P. D. (1984). On the average value of correlated time series, with applications in dendroclimatology and hydrometeorology. Journal of Climate and Applied Meteorology, 23(2), 201-213. doi: 10.1175/15200450(1984)023<0201:OTAVOC>2.0.CO;2.

Williams, A. P., Allen, C. D., Macalady, A. K., Griffin, D., Woodhouse, C. A., Meko, D. M., \& McDowell, N. G. (2013). Temperature as a potent driver of regional forest drought stress and tree mortality. Nature Climate Change, 3, 292-297. doi: 10.1038/nclimate1693

Wong, C. M., \& Daniels, L. D. (2017). Novel forest decline triggered by multiple interactions among climate, an introduced pathogen and bark beetles. Global Change Biology, 23, 1926-1941. doi: 10.1111/gcb.13554.

Worrall, J. J., Rehfeldt, G. E., Hamann, A., Hogg, E. H., Marchetti, S. B., Michaelian, M., \& Gray, L. K. (2013). Recent declines of Populus tremuloides in North America linked to climate. Forest Ecology and Management, 299, 35-51. doi: 10.1016/j.foreco.2012.12.033.

Manuscrito recibido el: 22 de junio de 2017

Aceptado el: 19 de octubre de 2017

Publicado el: 17 de mayo de 2018

\section{Este documento se debe citar como:}

Rodríguez-Catón, M., \& Villalba, R. (2018). Indicadores del decaimiento en bosques de Nothofagus pumilio en el norte de la Patagonia, Argentina. Madera y Bosques, 24(2), e2421588. doi: $10.21829 / \mathrm{myb} .2018 .2421588$

Madera y Bosques por Instituto de Ecología, A.C. se distribuye bajo una Licencia Creative Commons Atribución-NoComercialCompartirlgual 4.0 Internacional. 